\title{
Efficacy and Safety of a Mixed Extract of Trigonella foenum-graecum Seed and Lespedeza cuneata in the Treatment of Testosterone Deficiency Syndrome: A Randomized, Double-Blind, Placebo-Controlled Clinical Trial
}

\author{
Hyun Jun Park ${ }^{1,2(\mathbb{D})}$, Kyeong Soo Lee ${ }^{3}$ (D) Eun Kyoung Lee ${ }^{3}$ (D) Nam Cheol Park $^{1,2(\mathbb{C})}$ \\ ${ }^{1}$ Department of Urology, Pusan National University School of Medicine, ${ }^{2}$ Medical Research Institute of Pusan National University Hospital, \\ Busan, ${ }^{3}$ DUHANBIO CO., Seoul, Korea
}

\begin{abstract}
Purpose: The aim of this study was to investigate the efficacy and safety of a mixed extract of Trigonella foenum-graecum seed and Lespedeza cuneata (TFGL) for the treatment of testosterone deficiency syndrome (TDS).

Materials and Methods: Patients were instructed to take a placebo or 200 mg TFGL capsule twice per day for 8 weeks. The primary efficacy variable was the change from baseline in the Aging Males' Symptoms scale (AMS), as well as levels of serum total and free testosterone. Secondary efficacy measurements included changes from baseline in the number of 'yes' answers on the Androgen Deficiency in the Aging Male (ADAM) questionnaire, levels of serum total cholesterol, high density lipoprotein cholesterol (HDL-C), low density lipoprotein cholesterol (LDL-C), triglyceride, all domain scores of the International Index of Erectile Function (IIEF), perceived stress scale-10 (PSS-10), as well as changes in body composition.

Results: The TFGL group exhibited a significant improvement in the AMS scores at 8 weeks, total testosterone at 8 weeks, and free testosterone at 4 and 8 weeks. At 4 weeks, 25\% of the TFGL group changed to negative in terms of ADAM scores and $34.1 \%$ of the TFGL group had negative scores at the end of the study. The TFGL group exhibited a significant improvement in total cholesterol, HDL-C, LDL-C, triglyceride, IIEF scores, and PSS-10 scores at 8 weeks.

Conclusions: The mixed extract of TFGL resulted in significant improvements in symptoms of TDS, as measured by the AMS, ADAM, PSS-10 and testosterone levels.
\end{abstract}

Keywords: Herbal medicine; Hypogonadism; Phytotherapy; Testosterone

This is an Open Access article distributed under the terms of the Creative Commons Attribution Non-Commercial License (http://creativecommons.org/licenses/by-nc/4.0) which permits unrestricted non-commercial use, distribution, and reproduction in any medium, provided the original work is properly cited.

\section{INTRODUCTION}

Testosterone deficiency syndrome (TDS) is 'a clinical and biochemical syndrome associated with advancing age and characterized by symptoms of a deficiency in serum testosterone levels' [1]. TDS can cause a significant decrease in quality of life and has many adverse effects on multiple organs in terms of men's health. In addition

Received: Dec 9, 2017 Revised: Jan 15, 2018 Accepted: Jan 29, 2018 Published online Mar 22, 2018

Correspondence to: Nam Cheol Park iD https://orcid.org/0000-0003-2735-9278

Department of Urology, Pusan National University School of Medicine, 179 Gudeok-ro, Seo-gu, Busan 49241, Korea.

Tel: +82-51-240-7349, Fax: +82-51-247-5443, E-mail: pnc@pusan.ac.kr 
to the detrimental effect of sexual functions, men with TDS tend to have increased waist circumference, hyperglycemia, hypertriglyceridemia, hyperlipidemia, and type 2 diabetes [2]. TDS can be treated with testosterone replacement therapy (TRT). TRT should be initiated on an individualized basis in TDS patients who have clinical signs and symptoms of testosterone deficiency if the benefits of treatment appear to outweigh the potential risks and after thorough discussion with the patient [3]. Various preparations of testosterone are currently available, and the majority of clinical data shows that TRT is safe and effective. There is currently no evidence that testosterone treatment increases the risk of prostate disease according to modern guidelines [1]. However, the fear of prostate cancer and the risk of erythrocytosis have become major reason for reluctant use of TRT in aging men. Furthermore, TRT does not improve the function of Leydig cells that produce testosterone, but merely replenishes the insufficient testosterone [1,3].

Despite the increasing availability of effective conventional medical treatments, plant-derived and herbomineral remedies continue to be a popular alternative for men seeking to improve sexual function $[4,5]$. However, limited clinical research has focused on the use of herbal medicine to improve male health, particularly to increase testosterone levels and support healthy sexual function.

Trigonella foenum-graecum Linn, also known as fenugreek, is an aromatic annual plant that found wild in Kashmir, Punjab, and the upper Gangetic plains, and is widely cultivated in many parts of India. It is used internally as an abortifacient, antispasmodic, appetite stimulant, blood cleanser, laxative, tonic, and expectorant [6]. The seeds contain diosgenin along with three minor steroidal saponins (similagenin, savsalpogenin, and yuccagenin), choline, trimethylamine (a sex hormone in frogs), vitamins (A, B2, B6, B12, D), lysine, ltryptophan rich proteins, mucilaginous fiber, coumarin, fenugreekine, nicotinic acid, sapogenins, phytic acid, scopletin and trigonelline, calcium, iron, $\beta$-carotene, and other vitamins and essential oils [7].

Previous studies have suggested that Trigonella foenum-graecum seed extract has positive effects on sexual health and quality of life, and that it demonstrates anabolic and androgenic activity in young patients [8]. It is believed that these positive effects are due to increased testosterone, and that Trigonella foenumgraecum seed extract can be an effective treatment for the TDS in aging men. The basis for this androgenic activity may be due to the fact that Trigonella foenum-graecum seeds contain soluble steroidal saponins, specifically furostanol glycosides, which are responsible for complexing cholesterol in the cell membrane [9,10]. Other studies have found that Trigonella foenumgraecum increases testosterone and free testosterone, suggesting that it may be an incomplete 5-alpha reductase and aromatase inhibitor [11]. In this study, we investigated the efficacy and safety of a mixed extract of Trigonella foenum-graecum seed and Lespedeza cuneata (TFGL) for the treatment of TDS.

\section{MATERIALS AND METHODS}

\section{Study design}

This was a double-blind, randomized, placebo-controlled, parallel-group study conducted in accordance with the Good Clinical Practices standards and in conformity with the ethical principles set out in the Declaration of Helsinki. Patients were recruited prospectively and consecutively between June 2015 and November 2015. Initially, eligible patients had a one-week treatment-free run-in period and were checked for adequacy for inclusion. Subsequently, patients were randomly assigned to receive investigational products (200 mg TFGL or placebo) twice per day for 8 weeks at least 30 minutes after food intake (Fig. 1). During the 8-week treatment period, patient tolerance and responses to the investigational drugs were assessed every 4 weeks, and follow-up contact was made 7 days after the 8-week treatment to evaluate any additional adverse events.

\section{Ethics statement}

This study was approved by the Institutional Review Board of Seoul National University Bundang Hospital (IRB NO: B-1505/299-001). The study was retrospectively registered in the NIH ClinicalTrials.gov (http://www. clinicaltrials.gov/) (NCT03057899). Written informed consent was obtained from each patient before randomization.

\section{Subjects}

Inclusion criteria were as follows: men aged over 40 years, with total scores on the Aging Males' Symptoms scale (AMS) questionnaire $\geq 27$; total serum cholesterol $<220 \mathrm{mg} / \mathrm{dL}$; and triglyceride 150-399 mg/dL. Men with the following conditions were excluded from the 

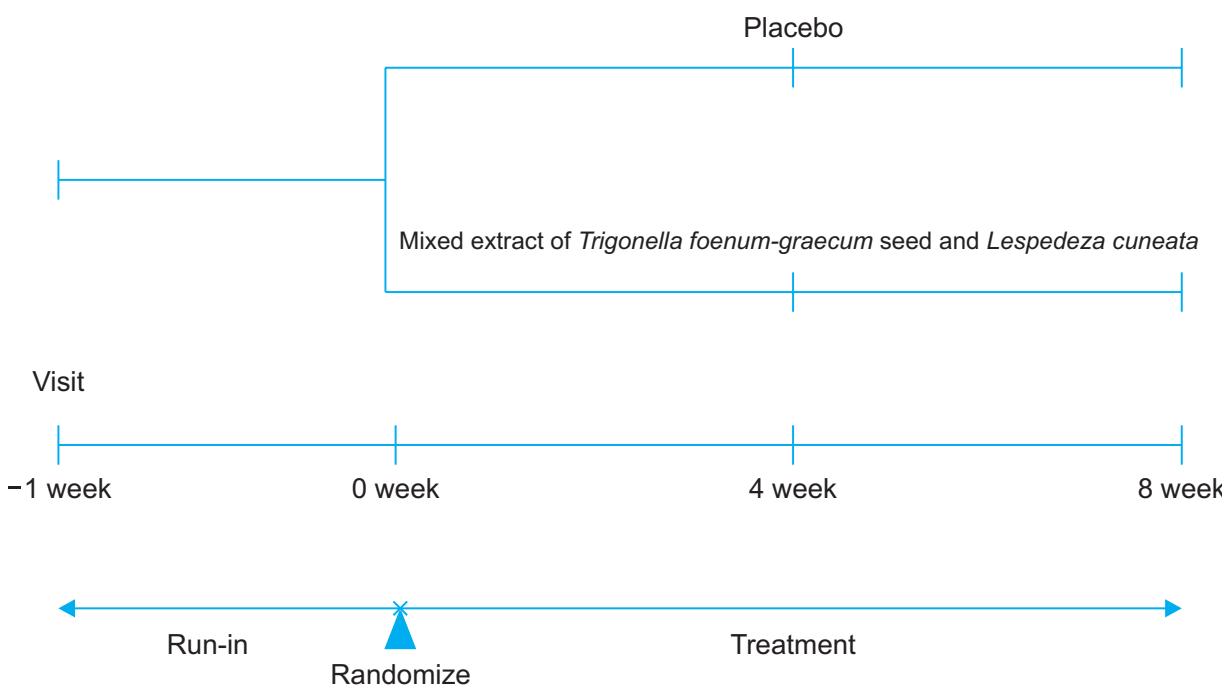

Fig. 1. Study design.

study: diagnosis of another sexual disorder, serum creatinine $>2.5 \mathrm{mg} / \mathrm{dL}$, an uncontrolled psychiatric disorder, history of major hematological, renal, or hepatic abnormalities, body mass index $\geq 45 \mathrm{~kg} / \mathrm{m}^{2}$, hepatitis B surface antigen positive, prostate specific antigen $\geq 4.0$ $\mathrm{ng} / \mathrm{mL}$, cardiac failure, or a history of alcoholism or substance abuse. Patients who had taken phosphodiesterase-5 (PDE-5) inhibitors, TRT, anti-androgen, statins, fibrates, niacin, steroid, fish oil, colestin, fiber-based laxatives, phytosterol margarines, anti-diabetics, antiplatelet, thyroxine, diuretics, or beta-blockers were also excluded. Concomitant use of any erectile dysfunction (ED) treatment was prohibited.

\section{Investigational drugs}

TFGL capsules (200 mg/capsule) and placebos were provided by DUHANBIO (Seoul, Korea). TFGL capsules were prepared using extracts from TFGL. During the study period, two capsules were taken daily for 8 weeks. Placebo and TFGL capsules were identical in shape, color, and taste.

\section{Outcome measures}

The primary efficacy variable was the change from baseline in the AMS [12] and serum levels of total testosterone and free testosterone. Secondary efficacy measurements included changes from baseline in percentage of positivity on the Androgen Deficiency in the Aging Males (ADAM) questionnaire [13], serum levels of total cholesterol, high density lipoprotein cholesterol (HDL-C), low density lipoprotein cholesterol (LDLC), triglyceride, all domain scores of the International
Index of Erectile Function (IIEF) [14], and perceived stress scale-10 (PSS-10) [15]. Changes in body composition were measured by using an InBody720 body composition analyser (InBody, Seoul, Korea). Adverse events were classified as adverse changes from baseline that occurred during the study period. Safety assessments included laboratory tests (hematology, clinical biochemistry, blood coagulation test, and urinalysis), vital signs (blood pressure and heart rate), physical examination, 12-lead electrocardiogram recordings, and patient-reported adverse events.

\section{Statistical methods}

Efficacy analysis was based on the full analysis set. Independent sample t-tests and repeated measures analysis of variance (ANOVA) were used to evaluate the primary efficacy endpoints and all secondary endpoints. Efficacy was calculated compared to the placebo group after assessing changes from baseline at given points, including the end of treatment. Statistical significance was set as $p<0.05$. The number of patients evaluated in this study was based on data obtained from the AMS from a previous study using a herbal formula [16]. According to the standards of a per-protocol analysis, 39 valid patients were required for each group. Assuming a 10\% dropout, 88 randomized patients (44 per group) were required for efficacy analysis. Changes from baseline in continuous safety variables, including laboratory analysis and vital signs, were evaluated by ANOVA. Between-group comparisons of treatment-emergent adverse event frequencies were conducted using $\chi^{2}$ test or Fisher's exact test. Baseline demographics in the TFGL 
and placebo groups were compared using ANOVA for continuous variables using the $\chi^{2}$ test for categorical variables. Block randomization was used and generated by the SAS Proc Plan procedure. Statistical analyses were performed using SAS ver. 9.1 (SAS Institute, Cary, NC, USA).

\section{RESULTS}

\section{Demographics}

In total, 88 men completed baseline evaluations and were randomly assigned to receive either placebo or $400 \mathrm{mg}$ TFGL. At baseline, no clinically or statistically significant differences were found between the treatment groups with respect to demographic or clinical variables (Table 1).

\section{Primary efficacy outcome variable}

\section{1) Aging Males' Symptoms scale}

In the analysis of differences, the TFGL group

Table 1. Baseline characteristics of patients

\begin{tabular}{lccc}
\hline \multicolumn{1}{c}{ Characteristic } & $\begin{array}{c}\text { TFGL } 400 \\
(\mathbf{n}=44)\end{array}$ & $\begin{array}{c}\text { Placebo } \\
(\mathbf{n}=44)\end{array}$ & p-value \\
\hline Age $(\mathrm{y})$ & $59.2 \pm 7.7$ & $57.0 \pm 8.4$ & 0.214 \\
Height $(\mathrm{cm})$ & $168.2 \pm 5.9$ & $170.1 \pm 4.7$ & 0.092 \\
Body weight $(\mathrm{kg})$ & $71.1 \pm 10.3$ & $70.2 \pm 7.4$ & 0.648 \\
BMI (kg/m $\left.{ }^{2}\right)$ & $25.1 \pm 3.2$ & $24.3 \pm 2.2$ & 0.146 \\
AMS & $38.3 \pm 11.3$ & $37.9 \pm 8.9$ & 0.868 \\
IIEF & $41.3 \pm 19.2$ & $40.7 \pm 17.9$ & 0.877 \\
ADAM questionnaire & $44(100)$ & $44(100)$ & - \\
$\quad$ positive rate & & & \\
PSS-10 & $16.9 \pm 4.2$ & $17.1 \pm 17.9$ & 0.934 \\
Serum total testosterone & $4.3 \pm 1.4$ & $4.4 \pm 1.4$ & 0.895 \\
$\quad$ (ng/mL) & & & \\
Serum free testosterone & $7.7 \pm 2.1$ & $7.7 \pm 2.2$ & 0.930 \\
$\quad$ (pg/mL) & & & \\
Serum total cholesterol & $182.3 \pm 26.2$ & $183.0 \pm 26.2$ & 0.900 \\
$\quad$ (mg/dL) & & & \\
Serum HDL-C (mg/dL) & $44.1 \pm 6.1$ & $44.0 \pm 6.2$ & 0.918 \\
Serum LDL-C (mg/dL) & $115.0 \pm 26.0$ & $117.7 \pm 21.3$ & 0.589 \\
Serum triglyceride & $219.0 \pm 55.3$ & $223.4 \pm 58.9$ & 0.717 \\
$\quad$ (mg/dL) & & & \\
\hline
\end{tabular}

Values are presented as mean \pm standard deviation or number (\%). $\mathrm{p}$ value indicates the independence sample t-test.

TFGL: Trigonella foenum-graecum seed and Lespedeza cuneate, BMI: body mass index, AMS: Aging Males' Symptoms scale, IIEF: International Index of Erectile Function, ADAM: Androgen Deficiency in the Aging Males, PSS-10: perceived stress scale-10, HDL-C: high density lipoprotein cholesterol, LDL-C: low density lipoprotein cholesterol. showed a statistically significant improvement regarding changes from baseline in AMS scores at 4 and 8 weeks. However, no significant improvements were observed in the placebo group. Based on a comparison of changes from baseline between the two groups, significant differences were observed at 8 weeks (Table 2 ).

\section{2) Serum total testosterone and free testosterone}

In the analysis of differences, the TFGL group exhibited a statistically significant improvement regarding changes from baseline in total testosterone and free testosterone at 4 and 8 weeks. However, no significant improvements were observed in the placebo group. Compared with the placebo group, significant differences were observed at 8 weeks in total testosterone and at 4 and 8 weeks in free testosterone (Table 2). In terms of changes from baseline to end point, there were increases in total testosterone $(0.54 \pm 0.85 \mathrm{ng} / \mathrm{mL})$ and free testosterone $(1.09 \pm 1.46 \mathrm{ng} / \mathrm{mL})$ in the TFGL group and decreases in total testosterone $(0.49 \pm 0.81 \mathrm{ng} / \mathrm{mL})$ and free testosterone $(0.77 \pm 1.16 \mathrm{ng} / \mathrm{mL})$ in the control group.

\section{Secondary efficacy outcome variable}

\section{1) Response to Androgen Deficiency in the Aging Males questionnaire}

At 4 weeks, $25 \%$ of the TFGL group changed to negative response to the ADAM questionnaire and 34.1\% responded negative at the end of study. However, no patients in the placebo group had negative response to the ADAM questionnaire at 4 or 8 weeks (Table 2).

\section{2) Serum total cholesterol, high density}

lipoprotein cholesterol, low density

lipoprotein cholesterol, and triglyceride

In the analysis of differences, the TFGL group showed a statistically significant improvement regarding changes from baseline in the total cholesterol, HDL-C, LDL-C, and triglyceride at 4 and 8 weeks. However, no significant improvements were observed in the placebo group. Compared with the placebo group, significant differences were observed in the TFGL group at 8 weeks in total cholesterol, and at 4 and 8 weeks in HDL-C, LDL-C, and triglyceride (Table 2).

In terms of changes from baseline to end point, the TFGL group showed decreases in total cholesterol $(9.66 \pm 13.70 \mathrm{mg} / \mathrm{dL}), \mathrm{LDL}-\mathrm{C}(13.23 \pm 21.66 \mathrm{mg} / \mathrm{dL})$, and triglyceride $(65.07 \pm 70.63 \mathrm{mg} / \mathrm{dL})$, and increases in HDL- 
Table 2. Effects of TFGL on investigated parameters

\begin{tabular}{|c|c|c|c|c|c|c|}
\hline Variable & Visit (wk) & TFGL $400(n=44)$ & $p$-value ${ }^{a}$ & Placebo $(n=44)$ & $p$-value ${ }^{a}$ & $p$-value ${ }^{b}$ \\
\hline \multirow[t]{3}{*}{ AMS } & 0 & $38.3 \pm 11.3$ & $<0.001$ & $37.9 \pm 8.9$ & 0.699 & 0.868 \\
\hline & 4 & $34.5 \pm 13.2$ & & $38.2 \pm 9.5$ & & 0.144 \\
\hline & 8 & $33.2 \pm 11.5$ & & $38.2 \pm 9.6$ & & 0.029 \\
\hline \multirow[t]{3}{*}{ IIEF } & 0 & $41.3 \pm 19.2$ & 0.015 & $40.7 \pm 17.9$ & 0.008 & 0.877 \\
\hline & 4 & $41.9 \pm 19.0$ & & $39.1 \pm 19.4$ & & 0.490 \\
\hline & 8 & $45.9 \pm 18.5$ & & $36.8 \pm 20.8$ & & 0.034 \\
\hline \multirow{3}{*}{$\begin{array}{l}\text { ADAM questionnaire } \\
\text { positive rate }\end{array}$} & 0 & $44(100)$ & & $44(100)$ & & - \\
\hline & 4 & $33(75.0)$ & & $44(100)$ & & $<0.001^{\mathrm{c}}$ \\
\hline & 8 & $29(65.9)$ & & $44(100)$ & & $<0.001^{c}$ \\
\hline \multirow[t]{3}{*}{ PSS-10 } & 0 & $16.9 \pm 4.3$ & 0.005 & $17.1 \pm 7.9$ & 0.017 & 0.934 \\
\hline & 4 & $15.4 \pm 4.4$ & & $17.3 \pm 7.7$ & & 0.170 \\
\hline & 8 & $14.8 \pm 5.1$ & & $17.8 \pm 7.9$ & & 0.038 \\
\hline \multirow{3}{*}{$\begin{array}{l}\text { Serum total testosterone } \\
(\mathrm{ng} / \mathrm{mL})\end{array}$} & 0 & $4.3 \pm 1.4$ & $<0.001$ & $4.4 \pm 1.4$ & $<0.001$ & 0.895 \\
\hline & 4 & $4.4 \pm 1.5$ & & $4.02 \pm 1.3$ & & 0.201 \\
\hline & 8 & $4.9 \pm 1.6$ & & $3.9 \pm 1.2$ & & 0.002 \\
\hline \multirow{3}{*}{$\begin{array}{l}\text { Serum free testosterone } \\
(\mathrm{pg} / \mathrm{mL})\end{array}$} & 0 & $7.7 \pm 2.1$ & $<0.001$ & $7.7 \pm 2.2$ & $<0.001$ & 0.930 \\
\hline & 4 & $8.5 \pm 2.2$ & & $7.4 \pm 1.8$ & & 0.022 \\
\hline & 8 & $8.8 \pm 2.2$ & & $6.9 \pm 1.7$ & & $<0.001$ \\
\hline \multirow{3}{*}{$\begin{array}{l}\text { Serum total cholesterol } \\
(\mathrm{mg} / \mathrm{dL})\end{array}$} & 0 & $182.3 \pm 26.2$ & $<0.001$ & $183.0 \pm 26.2$ & $<0.001$ & 0.900 \\
\hline & 4 & $180.6 \pm 28.8$ & & $186.9 \pm 26.4$ & & 0.285 \\
\hline & 8 & $172.6 \pm 27.3$ & & $190.5 \pm 26.1$ & & 0.002 \\
\hline \multirow[t]{3}{*}{ Serum HDL-C (mg/dL) } & 0 & $44.1 \pm 6.1$ & $<0.001$ & $44.0 \pm 6.2$ & $<0.001$ & 0.918 \\
\hline & 4 & $46.8 \pm 8.6$ & & $42.6 \pm 7.0$ & & 0.014 \\
\hline & 8 & $49.2 \pm 8.7$ & & $40.2 \pm 6.7$ & & $<0.001$ \\
\hline \multirow[t]{3}{*}{ Serum LDL-C (mg/dL) } & 0 & $115.0 \pm 26.0$ & $<0.001$ & $117.7 \pm 21.3$ & $<0.001$ & 0.589 \\
\hline & 4 & $108.2 \pm 24.2$ & & $121.2 \pm 20.4$ & & 0.008 \\
\hline & 8 & $101.7 \pm 21.1$ & & $125.4 \pm 21.3$ & & $<0.001$ \\
\hline \multirow{3}{*}{$\begin{array}{l}\text { Serum triglyceride } \\
(\mathrm{mg} / \mathrm{dL})\end{array}$} & 0 & $219.0 \pm 55.3$ & $<0.001$ & $223.4 \pm 58.9$ & 0.011 & 0.717 \\
\hline & 4 & $175.0 \pm 58.2$ & & $231.6 \pm 53.0$ & & $<0.001$ \\
\hline & 8 & $153.9 \pm 61.8$ & & $238.5 \pm 59.9$ & & $<0.001$ \\
\hline \multirow{3}{*}{$\begin{array}{l}\text { Skeletal muscle } \\
\text { mass }(\mathrm{kg})\end{array}$} & 0 & $28.8 \pm 3.8$ & 0.066 & $29.2 \pm 3.6$ & 0.658 & 0.581 \\
\hline & 4 & $28.9 \pm 3.8$ & & $29.2 \pm 3.5$ & & 0.764 \\
\hline & 8 & $29.0 \pm 3.7$ & & $29.2 \pm 3.5$ & & 0.720 \\
\hline \multirow[t]{3}{*}{ Fat mass $(\mathrm{kg})$} & 0 & $18.4 \pm 4.6$ & 0.237 & $18.6 \pm 6.3$ & 0.773 & 0.860 \\
\hline & 4 & $18.1 \pm 4.5$ & & $18.7 \pm 6.4$ & & 0.640 \\
\hline & 8 & $18.4 \pm 4.5$ & & $18.6 \pm 6.2$ & & 0.827 \\
\hline
\end{tabular}

Values are presented as mean \pm standard deviation or number (\%).

TFGL: Trigonella foenum-graecum seed and Lespedeza cuneate, AMS: Aging Males' Symptoms scale, IIEF: International Index of Erectile Function, ADAM: Androgen Deficiency in the Aging Males, PSS-10: perceived stress scale-10, HDL-C: high density lipoprotein cholesterol, LDL-C: low density lipoprotein cholesterol.

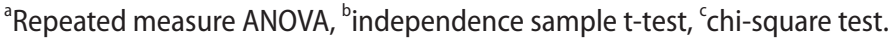

C $(5.02 \pm 5.72 \mathrm{mg} / \mathrm{dL})$. In contrast, the placebo group showed increases in total cholesterol $(7.57 \pm 10.40 \mathrm{mg} / \mathrm{dL})$, LDL-C (7.73 $\pm 12.79 \mathrm{mg} / \mathrm{dL})$, and triglyceride $(15.11 \pm 40.38$ $\mathrm{mg} / \mathrm{dL})$, and decreases in HDL-C $(3.77 \pm 4.75 \mathrm{mg} / \mathrm{dL})$.

\section{3) International Index of Erectile Function}

In the analysis of differences, the TFGL group showed a statistically significant improvement regarding changes from baseline in IIEF scores at 4 and 8 weeks. However, no significant improvements were observed in the placebo group. Compared with the placebo group, significant differences were observed in the TFGL group 
at 8 weeks (Table 2). In terms of changes from baseline to end point, there was an increase in IEF scores (4.66 \pm 10.30$)$ in the TFGL group and a dcrease (3.82 \pm 9.15$)$ in the placebo group.

\section{4) Perceived stress scale-10 questionnaire}

In the analysis of differences, the TFGL group exhibited statistically significant improvements regarding changes from baseline in PSS-10 scores at 4 and 8 weeks. However, it was rather deteriorated in the placebo group (Table 2).

\section{5) Body composition (skeletal muscle mass and fat mass)}

No significant changes were observed in body composition (skeletal muscle mass and fat mass) in either group at 4 or 8 weeks (Table 2).

\section{Safety and tolerability}

The safety analysis of TFGL included 44 subjects who took at least one dose. No clinically significant changes in laboratory tests, electrocardiogram, or blood pressure were observed in either group.

\section{DISCUSSION}

Aphrodisiac properties of Trigonella foenum-graecum (fenugreek) seed have been reported in ethnobotanical literature. Traditional Chinese herbalists used Trigonella foenum-graecum for male reproductive issues [17]. Trigonella foenum-graecum seed is also believed to have restorative and nutritive properties and to stimulate digestive processes [18]. Furostanol glycosides from a variety of plants have been shown to have adaptogenic, anabolic, or androgenic activity, especially as vitalizers to improve sexual function in men [19] and these effects are attributed mainly to protodioscin and related compounds [20]. In addition, diosgenin (an important precursor for the synthesis of a number of sex hormones including testosterone and estrogens) and saponins (especially protodioscin-like compounds) are reportedly present in Trigonella foenum-graecum seed [21,22]. Therefore, a glycoside-rich fraction of Trigonella foenum-graecum seed is worth investigating for possible androgenic and anabolic activity.

We already reported that 4 weeks of TFGL ( $40 \mathrm{mg} /$ $\mathrm{kg}$ and $80 \mathrm{mg} / \mathrm{kg}$ ) administration increased the serum testosterone levels, vastus lateralis muscular strength, forced swimming time, total sperm counts, and motile sperm counts in rats. Moreover, sex hormone binding globulin, the epididymal fat pad, total cholesterol, and triglyceride levels were significantly decreased in the TFGL-fed rats [23]. The dosage of the investigational drug used in this study was determined by converting the dosages that were confirmed to be efficient by the authors' previous in vitro studies [23,24].

In this study, we found that Trigonella foenum-graecum improved TDS symptoms and increased serum total testosterone and free testosterone. Erectile function measured by the IIEF, also increased in the treatment group. Our results are supported by those of two previous studies. One study focused on younger men using the same Trigonella foenum-graecum seed extract, which was found to have a positive effect on sexual function in men experiencing low libido [8]. In the other study, Rao et al [9] found that Testofen, a specialized Trigonella foenum-graecum seed extract, reduced age-related symptoms of androgen decrease, increased testosterone levels, and improved sexual function in healthy aging males in a double-blind randomized clinical study. An animal study by Hamden et al [25] found that daily oral treatment of fenugreek steroids to diabetic rats over 30 days induced a considerable increase in testosterone in the plasma of the rats. However, a clinical study by Steels et al [8] and an animal study using male albino rats conducted by Aswar et al [26] did not reveal significant changes in testosterone levels.

We hypothesize that the reduction in symptoms of TDS and increased sexual function was directly or indirectly related to the increased serum testosterone. Potential mechanisms by which Trigonella foenumgraecum may increase serum testosterone include stimulation of pulsatile gonadotropin-releasing hormone $(\mathrm{GnRH}) /$ luteinizing hormone $(\mathrm{LH})$, increased testicular sensitivity to $\mathrm{LH}$, and increased testosterone synthesis or reduced testosterone catabolism. However, further studies are required before a clear mechanism of action can be proposed. We demonstrated that Trigonella foenum-graecum improved lipid profiles, including decreases in total cholesterol, LDL-C, and triglyceride, as well as increases in HDL-C. These findings are consistent with earlier studies using experimentally induced hyperlipidemia in rabbits $[27,28]$. The mechanism of antihyperlipidemic activities of Trigonella foenumgraecum remains unclear, but it has been suggested 
that total dietary fiber may play a key role in reducing cholesterol levels through increased fecal excretion of bile acids and salts, as well as inhibiting hepatic cholesterol biosynthesis by short chain fatty acids produced by bacterial fermentation of soluble dietary fiber in the lower parts of the large intestine [28].

With regard to body composition, we found no significant changes (in skeletal muscle mass and fat mass) in either group at 4 or 8 weeks. However, Aswar et al [26] reported that Trigonella foenum-graecum increased the weight of the levator ani muscle, as well as the body weight of castrated rats. They suggested that Trigonella foenum-graecum has anabolic properties (increasing muscle mass), and that the probable mechanism for this action is increased availability of testosterone by dissociating it from the stored form, i.e., sex hormone-binding globulin (SHBG). Further studies are required to explore the effects of Trigonella foenum-graecum on body composition.

We used L. cuneata as a mixing ingredient. L. cuneate has been used therapeutically in traditional Asian medicine to protect the function of liver, kidneys, and lungs. Kim et al [29] observed the hepatoprotective effects of L. cuneata and found a high correlation with radical scavenging activity, which followed the structure-activity relationships of the flavonoid aglycones. Based on an in vitro study, Lee et al [30] reported that $L$. cuneate dilated vascular smooth muscle via endothelium-dependent nitric oxide-cyclic guanosine monophosphate (NO-cGMP) signaling, which is a similar mechanism of PDE-5 inhibitors for treating ED.

The present study had several limitations. Although we observed significant improvements in the AMS domain as the primary outcome variable, as well as some secondary outcome variables, we did not observe statistically significant improvements in other parameters such as body composition. This may have been due to the short study duration, which was not sufficient to change body composition. Second, due to sociocultural differences among various populations of different ethnic origins, the efficacy and safety profile of Trigonella foenum-graecum observed in this study, which included only Korean patients, may mean that outcomes differ somewhat across different ethnic groups. A third limitation is that this study did not use a cross-over design. Larger clinical trials will be required to investigate the efficacy and safety of Trigonella foenum-graecum in other ethnicities and TDS of various causes, using dif- ferent dosing regimens and direct comparative studies with testosterone. Lastly, we evaluated only total scores of AMS and IIEF. An analysis of each domain of AMS and IIEF would be beneficial to clarify changes in the symptoms of TDS.

\section{CONCLUSIONS}

In summary, a mixed extract of TFGL significantly improved symptoms of TDS, as measured by the AMS, ADAM, PSS-10 and testosterone increase. With TRT already in clinical use, a herbal formula of TFGL has the potential to expand treatment options for TDS.

\section{Disclosure}

Dr. Eun Kyoung Lee and Kyeong Soo Lee are employee of the DUHANBIO Pharmaceutical Co. Ltd., Seoul, Korea, but they made no influence on this work. Other authors have no potential conflicts of interest to disclose.

\section{Author Contribution}

Research conception \& design: Lee KS, Lee EK, Park NC. Performing the experiments: Lee KS, Lee EK, Park NC. Data acquisition: Lee KS, Lee EK. Data analysis and interpretation: Park HJ, Park NC. Statistical analysis: Park HJ, Park NC. Drafting of the manuscript: Park HJ, Park NC. Critical revision of the manuscript: Park HJ, Park NC. Approval of final manuscript: all authors.

\section{REFERENCES}

1. Dean JD, McMahon CG, Guay AT, Morgentaler A, Althof SE, Becher EF, et al. The International Society for sexual medicine's process of care for the assessment and management of testosterone deficiency in adult men. J Sex Med 2015;12:166086.

2. Tsujimura A. The relationship between testosterone deficiency and men's health. World J Mens Health 2013;31:126-35.

3. Corona G, Sforza A, Maggi M. Testosterone replacement therapy: long-term safety and efficacy. World J Mens Health 2017;35:65-76.

4. Aung HH, Dey L, Rand V, Yuan CS. Alternative therapies for male and female sexual dysfunction. Am J Chin Med 2004; 32:161-73.

5. Moon HW, Park JW, Lee KW, Jeong HC, Choi JB, Choi SW, et al. Administration of Goji (Lycium chinense Mill.) extracts 
improves erectile function in old aged rat model. World J Mens Health 2017;35:43-50.

6. Ziyyat A, Legssyer A, Mekhfi H, Dassouli A, Serhrouchni M, Benjelloun W. Phytotherapy of hypertension and diabetes in oriental Morocco. J Ethnopharmacol 1997;58:45-54.

7. Yadav SK, Sehgal S. Effect of home processing and storage on ascorbic acid and beta-carotene content of Bathua (Chenopodium album) and fenugreek (Trigonella foenum graecum) leaves. Plant Foods Hum Nutr 1997;50:239-47.

8. Steels E, Rao A, Vitetta L. Physiological aspects of male libido enhanced by standardized Trigonella foenum-graecum extract and mineral formulation. Phytother Res 2011;25:1294300.

9. Rao A, Steels E, Inder WJ, Abraham S, Vitetta L. Testofen, a specialised Trigonella foenum-graecum seed extract reduces age-related symptoms of androgen decrease, increases testosterone levels and improves sexual function in healthy aging males in a double-blind randomised clinical study. Aging Male 2016;19:134-42.

10. Mohanty PP, Naveh-Benjamin M, Ratneshwar S. Beneficial effects of semantic memory support on older adults' episodic memory: differential patterns of support of item and associative information. Psychol Aging 2016;31:25-36.

11. Wilborn C, Taylor L, Poole C, Foster C, Willoughby D, Kreider R. Effects of a purported aromatase and $5 a$-reductase inhibitor on hormone profiles in college-age men. Int J Sport Nutr Exerc Metab 2010;20:457-65.

12. Heinemann LA, Saad F, Zimmermann T, Novak A, Myon E, Badia X, et al. The Aging Males' Symptoms (AMS) scale: update and compilation of international versions. Health Qual Life Outcomes 2003;1:15.

13. Morley JE, Perry HM 3rd. Androgen deficiency in aging men: role of testosterone replacement therapy. J Lab Clin Med 2000;135:370-8.

14. Rosen RC, Riley A, Wagner G, Osterloh IH, Kirkpatrick J, Mishra A. The international index of erectile function (IIEF): a multidimensional scale for assessment of erectile dysfunction. Urology 1997;49:822-30.

15. Lee EH, Chung BY, Suh CH, Jung JY. Korean versions of the Perceived Stress Scale (PSS-14, 10 and 4): psychometric evaluation in patients with chronic disease. Scand J Caring Sci 2015;29:183-92.

16. Udani JK, George AA, Musthapa M, Pakdaman MN, Abas A. Effects of a Proprietary Freeze-Dried Water Extract of Eurycoma longifolia (Physta) and Polygonum minus on sexual performance and well-being in men: a randomized, doubleblind, placebo-controlled study. Evid Based Complement Alternat Med 2014;2014:179529.
17. Basch E, Ulbricht C, Kuo G, Szapary P, Smith M. Therapeutic applications of fenugreek. Altern Med Rev 2003;8:20-7.

18. Pandey H, Awasthi P. Effect of processing techniques on nutritional composition and antioxidant activity of fenugreek (Trigonella foenum-graecum) seed flour. J Food Sci Technol 2015;52:1054-60.

19. Park SW, Lee CH, Shin DH, Bang NS, Lee SM. Effect of SA1, a herbal formulation, on sexual behavior and penile erection. Biol Pharm Bull 2006;29:1383-6.

20. Gauthaman K, Ganesan AP, Prasad RN. Sexual effects of puncturevine (Tribulus terrestris) extract (protodioscin): an evaluation using a rat model. J Altern Complement Med 2003;9:257-65.

21. Aradhana, Rao AR, Kale RK. Diosgenin: a growth stimulator of mammary gland of ovariectomized mouse. Indian J Exp Biol 1992;30:367-70.

22. Hibasami H, Moteki H, Ishikawa K, Katsuzaki H, Imai K, Yoshioka K, et al. Protodioscin isolated from fenugreek (Trigonella foenumgraecum L.) induces cell death and morphological change indicative of apoptosis in leukemic cell line H-60, but not in gastric cancer cell line KATO III. Int J Mol Med 2003;11:23-6.

23. Lee KS, Lee EK, Kim SY, Kim TH, Kim HP. Effect of a mixed extract of fenugreek seeds and Lespedeza cuneata on testosterone deficiency syndrome. Korean J Food Sci Technol 2015; 47:492-8.

24. USFDA. Guidance for industry: estimating the maximum safe starting dose in adult healthy volunteer. Rockville, MD: US Food and Drug Administration; 2005.

25. Hamden K, Jaouadi B, Carreau S, Aouidet A, El-Fazaa S, Gharbi N, et al. Potential protective effect on key steroidogenesis and metabolic enzymes and sperm abnormalities by fenugreek steroids in testis and epididymis of surviving diabetic rats. Arch Physiol Biochem 2010;116:146-55.

26. Aswar U, Bodhankar SL, Mohan V, Thakurdesai PA. Effect of furostanol glycosides from Trigonella foenum-graecum on the reproductive system of male albino rats. Phytother Res 2010;24:1482-8.

27. Sharma MS, Choudhary PR. Hypolipidemic effect of fenugreek seeds and its comparison with atorvastatin on experimentally induced hyperlipidemia. J Coll Physicians Surg Pak 2014;24:539-42.

28. Sharma MS, Choudhary PR. Effect of fenugreek seeds powder (Trigonella foenum-graecum L.) on experimental induced hyperlipidemia in rabbits. J Diet Suppl 2016. doi: 10.3109/19390211.2016.1168905 [Epub].

29. Kim SM, Kang K, Jho EH, Jung YJ, Nho CW, Um BH, et al. Hepatoprotective effect of flavonoid glycosides from Lespe- 
deza cuneata against oxidative stress induced by tert-butyl hyperoxide. Phytother Res 2011;25:1011-7.

30. Lee JK, Kang DG, Lee HS. Vascular relaxation induced by aqueous extract of Lespedeza cuneata via the NO-cGMP pathway. J Nat Med 2012;66:17-24. 